\title{
Dependence of two-proton radioactivity on nuclear pairing models
}

\section{Oishi, Tomohiro}

2017-10-26

Oishi , T , Kortelainen , M \& Pastore , A 2017 , ' Dependence of two-proton radioactivity on nuclear pairing models ' , Physical Review C , vol. 96 , no. 4 , 044327 . https://doi.org/10.1103/PhysRevC.96.044327

http://hdl.handle.net/10138/231679

https://doi.org/10.1103/PhysRevC.96.044327

publishedVersion

Downloaded from Helda, University of Helsinki institutional repository.

This is an electronic reprint of the original article.

This reprint may differ from the original in pagination and typographic detail.

Please cite the original version. 


\title{
Dependence of two-proton radioactivity on nuclear pairing models
}

\author{
Tomohiro Oishi, ${ }^{1,2,3,{ }^{*}}$ Markus Kortelainen, ${ }^{1,2}$ and Alessandro Pastore ${ }^{4}$ \\ ${ }^{1}$ Helsinki Institute of Physics, P.O. Box 64, FI-00014 University of Helsinki, Helsinki, Finland \\ ${ }^{2}$ Department of Physics, P.O. Box 35 (YFL), University of Jyvaskyla, FI-40014 Jyvaskyla, Finland \\ ${ }^{3}$ Research Center for Electron Photon Science, Tohoku University, 1-2-1 Mikamine, Sendai 982-0826, Japan \\ ${ }^{4}$ Department of Physics, University of York, Heslington, York YO10 5DD, United Kingdom \\ (Received 14 June 2016; revised manuscript received 5 May 2017; published 26 October 2017)
}

\begin{abstract}
Sensitivity of two-proton emitting decay to nuclear pairing correlation is discussed within a time-dependent three-body model. We focus on the ${ }^{6} \mathrm{Be}$ nucleus assuming $\alpha+p+p$ configuration, and its decay process is described as a time evolution of the three-body resonance state. For a proton-proton subsystem, a schematic density-dependent contact (SDDC) pairing model is employed. From the time-dependent calculation, we observed the exponential decay rule of a two-proton emission. It is shown that the density dependence does not play a major role in determining the decay width, which can be controlled only by the asymptotic strength of the pairing interaction. This asymptotic pairing sensitivity can be understood in terms of the dynamics of the wave function driven by the three-body Hamiltonian, by monitoring the time-dependent density distribution. With this simple SDDC pairing model, there remains an impossible trinity problem: it cannot simultaneously reproduce the empirical $Q$ value, decay width, and the nucleon-nucleon scattering length. This problem suggests that a further sophistication of the theoretical pairing model is necessary, utilizing the two-proton radioactivity data as the reference quantities.
\end{abstract}

DOI: 10.1103/PhysRevC.96.044327

\section{INTRODUCTION}

Description of nuclear pairing correlation has been a major subject in recent nuclear physics. For instance, in selfconsistent meanfield (SCMF) description of atomic nuclei, there have been various approaches in order to take the nuclear pairing correlation into account [1-6]. These approaches based on the SCMF or the nuclear density functional theory (DFT) have provided considerable agreement with the measured binding energy as well as its odd-even staggering for bound nuclei. Even with these efforts, however, the complete character of the nuclear pairing correlation has not been revealed. For example, whether the phenomenological pairing interaction should have the volume or surface type of the density dependence is still an open question [7-10]. At present, one can find several candidates for the sophisticated nuclear pairing model [7-12]. In order to validate these models, one may need reference observables to fit not only for bound nuclei. Also, it should be emphasized that the pairing correlation near and beyond the neutron and proton drip lines could play a fundamental role to determine the limit of existence on the nuclear chart [13,14].

Recently, it has been expected that the two-proton $(2 p)$ radioactivity may provide novel information on the nuclear pairing correlation. In the true $2 p$ emission [15-20], a pair of protons is emitted simultaneously from the parent nucleus, whereas the single-proton emission is forbidden or strongly suppressed due to the pairing correlation energy. The proton-proton pairing plays an essential role to determine the observables, especially the released energy ( $Q$ value) as well

\footnotetext{
*Present address: Department of Physics and Astronomy "Galileo Galilei," University of Padova, Italy; toishi@pd.infn.it
}

as the $2 p$-decay width or lifetime [19-26]. The released $Q$ value can be related to the proton-proton pairing strength in bound nuclei. On the other hand, the $2 p$-decay width has no correspondence in bound systems, whose lifetime is trivially infinite. Thus, $2 p$ decays may provide another lodestar with new experimental input to optimize and validate the pairing models. Thanks to the experimental developments, there has been a considerable accumulation of data for the $2 p$-emitting nuclei [18-20]. On the other side, however, theoretical studies have not been sufficient to clarify the relation between the $2 p$ radioactivity and the pairing correlation. Because $2 p$ emission is a typical many-body problem, its elucidation could also provide a universal knowledge on the multiparticle quantum phenomena in various domains. Those include, e.g., the quantum entanglement [27], BCS-BEC crossover [28,29], and Efimov physics [30-32].

In this article, we discuss how the theoretical characters of pairing models are reflected on $2 p$-decay properties, connected to a specific interest in sophisticating those models. For this purpose, we employ the time-dependent three-body model [33], whose simplicity enables us to phenomenologically understand the pairing model dependence of $2 p$ radioactivity. We focus on the $2 p$ emission from the ground state of the ${ }^{6} \mathrm{Be}$ nucleus, assuming the configuration of two valence protons and a rigid $\alpha$ core.

The formalism of our model is given in Sec. II. In Sec. III, we present numerical calculations and discussions. Finally Sec. IV is devoted to summarize the article.

\section{THREE-BODY MODEL}

Details of the time-dependent three-body calculations are present in Ref. [33]. In this article, we employ this method but with some changes. The $2 p$ decay from ${ }^{6} \mathrm{Be}$ is described as a 
time evolution of the two protons in the spherical mean field generated by the $\alpha$ core. The three-body Hamiltonian is given as [29,34-37]

$$
H_{3 \mathrm{~b}}=h_{1}+h_{2}+\frac{\boldsymbol{p}_{1} \cdot \boldsymbol{p}_{2}}{A_{\mathrm{c}} m}+v_{\mathrm{p}-\mathrm{p}}\left(\boldsymbol{r}_{1}, \boldsymbol{r}_{2}\right),
$$

where $h_{i}=p_{i}^{2} / 2 \mu+V_{\mathrm{c}-\mathrm{p}}\left(r_{i}\right)(i=1,2)$ is the single particle (s.p.) Hamiltonian between the core and the $i$ th proton. $\mu \equiv$ $m A_{\mathrm{c}} /\left(A_{\mathrm{c}}+1\right)$ is the reduced s.p. mass with $A_{\mathrm{c}}=4$.

The core-proton potential is given as $V_{\mathrm{c}-\mathrm{p}}(r)=V_{\mathrm{WS}}(r)+$ $V_{\text {Coul }}(r)$. The Woods-Saxon potential is expressed as

$$
V_{\mathrm{WS}}(r)=V_{0} f(r)+U_{l s}(\boldsymbol{l} \cdot \boldsymbol{s}) \frac{1}{r} \frac{d f(r)}{d r},
$$

with a function

$$
f(r)=\frac{1}{1+e^{\left(r-R_{f}\right) / a_{f}}},
$$

where $R_{f}=1.68 \mathrm{fm}$ and $a_{f}=0.615 \mathrm{fm}$. $V_{\text {Coul }}$ describes the Coulomb potential. For $V_{\mathrm{WS}}(r)$ and $V_{\mathrm{Coul}}(r)$, we employ the same parameters as in Ref. [33], in order to reproduce the resonance energy and width in the $\left(p_{3 / 2}\right)$ channel of $\alpha-p$ scattering, $\epsilon_{r}=1.96 \mathrm{MeV}$ and $\Gamma_{r} \simeq 1.5 \mathrm{MeV}$, respectively [38]. Note that this resonance is attributable to the centrifugal potential barrier [33].

The $p$ - $p$ pairing potential is introduced as $v_{\mathrm{p}-\mathrm{p}}=v_{\mathrm{p}-\mathrm{p}}^{(N)}+$ $e^{2} /\left|\boldsymbol{r}_{1}-\boldsymbol{r}_{2}\right|$. Here, we employ a schematic density-dependent contact (SDDC) potential,

$$
\begin{aligned}
v_{\mathrm{p}-\mathrm{p}}^{(N)}\left(\boldsymbol{r}_{1}, \boldsymbol{r}_{2}\right) & =w\left[\left|\left(\boldsymbol{r}_{1}+\boldsymbol{r}_{2}\right) / 2\right|\right] \delta\left(\boldsymbol{r}_{1}-\boldsymbol{r}_{2}\right), \\
w(r) & =w_{0}[1-\eta f(r)],
\end{aligned}
$$

for the nuclear pairing interaction: two protons have a contact pairing correlation, whose density dependence is schematically approximated as the $\eta f(r)$ term. For the sake of generality, the density dependence is not limited to have the same $f(r)$ in Eqs. (2) and (4). In this work, however, we use the same function for simplicity, except in Sec. III D. Notice also that $w(r \longrightarrow \infty)=w_{0}$. For intrinsic two-nucleon structures, including the dinucleon correlation, similar three-body model calculations with SDDC pairing models have been utilized [29,34-37], with a consistency between other theoretical results [39-42].

With the contact interaction, it is generally known that one should introduce the energy cutoff $E_{\text {cut }}$ in order to avoid the ultraviolet divergence [43]. In the present case, the bare strength $w_{0}$ can be determined so as to reproduce the empirical scattering length of nucleons in vacuum, $a_{0}=-18.5 \mathrm{fm}$, consistently to the energy cutoff $[34,35]$. That is,

$$
w_{0}=\frac{4 \pi^{2} \hbar^{2} a_{0}}{m\left(\pi-2 a_{0} k_{\mathrm{cut}}\right)},
$$

with $k_{\text {cut }}=\sqrt{m E_{\text {cut }}} / \hbar$. The cutoff energy is set as $E_{\text {cut }}=40 \mathrm{MeV}$ similarly as in Ref. [33], yielding $w_{0}=$ $-767.398 \mathrm{MeV} \mathrm{fm}^{3}$.

Total expectation value of $H_{3 \mathrm{~b}}$, which is conserved during the time evolution, corresponds to the released $Q$ value of the three-body decay. That is,

$$
\begin{aligned}
Q_{2 \mathrm{p}} & =\left\langle\Psi(t)\left|H_{3 \mathrm{~b}}\right| \Psi(t)\right\rangle, \\
& =\left\langle h_{1}+h_{2}\right\rangle_{(t)}+\Delta_{\text {pair }}(t), \\
\Delta_{\text {pair }}(t) & =\left\langle\Psi(t)\left|\frac{\boldsymbol{p}_{1} \cdot \boldsymbol{p}_{2}}{A_{\mathrm{c}} m}+v_{\mathrm{p}-\mathrm{p}}\left(\boldsymbol{r}_{1}, \boldsymbol{r}_{2}\right)\right| \Psi(t)\right\rangle,
\end{aligned}
$$

where $\Delta_{\text {pair }}$ is the pairing correlation energy (PCE). In order to reproduce the empirical $Q$ value, $1371 \pm 5 \mathrm{keV}$ [38], we should employ a density-dependence parameter, $\eta=1.04$, in Eq. (4). Namely, the empirical $Q$ value requires almost the surface type of the SDDC pairing interaction, which imitates the surface version of the density-dependent pairing energy in DFT calculations [7-9]. Note also that PCE approximately corresponds to the pairing gap when the system is bound.

We assume the $0^{+}$configuration for two protons and the $\alpha$ core, consistently with the total spin parity which is also $0^{+}$for the ground state of ${ }^{6} \mathrm{Be}$. Thus, the eigenstates of the three-body Hamiltonian, satisfying $H_{3 \mathrm{~b}}\left|E_{N}\right\rangle=E_{N}\left|E_{N}\right\rangle$, can be expanded on the $0^{+}$-configuration basis:

$$
\begin{aligned}
\left|E_{N}\right\rangle & =\sum_{M} U_{N M}\left|\Phi_{M}\right\rangle, \\
\Phi_{M}\left(\boldsymbol{r}_{1}, \boldsymbol{r}_{2}\right) & =\hat{\mathcal{A}}\left[\phi_{n_{a} l j m}\left(\boldsymbol{r}_{1}\right) \otimes \phi_{n_{b} l j(-m)}\left(\boldsymbol{r}_{2}\right)\right]^{0^{+}},
\end{aligned}
$$

with the simplified notation, $M=\left(n_{a}, n_{b}, l, j\right)$. Here $\hat{\mathcal{A}}$ is the antisymmetrization operator. The expansion coefficients $U_{N M}$ are determined by diagonalizing the Hamiltonian matrix for $H_{3 \mathrm{~b}}$. Each s.p. state satisfies $h_{i} \phi_{n l j m}\left(\boldsymbol{r}_{i}\right)=\epsilon_{n l j} \phi_{n l j m}\left(\boldsymbol{r}_{i}\right)$, with the radial quantum number $n$, the orbital angular momentum $l$, the spin-coupled angular momentum $j$, and the magnetic quantum number $m$. We employ the s.p. states up to the $\left(h_{11 / 2}\right)$ channel. In order to take into account the Pauli principle, we exclude the first $s_{1 / 2}$ state, which is occupied by the protons in the core nucleus. The continuum s.p. states $\left(\epsilon_{n l j}>0\right)$ of $V_{\text {c-p }}$ are discretized in the radial box of $R_{\mathrm{box}}=80 \mathrm{fm}$. Thus, continuum eigenstates of $H_{3 \mathrm{~b}}$ are also discretized. As we present in Sec. IIIC, this radial box is sufficiently large to provide a good convergence in terms of the decay width.

It is worthwhile to mention that, if one can neglect PCE, a $2 p$-resonance state locates at $Q_{2 \mathrm{p}}=\left\langle h_{1}+h_{2}\right\rangle=2 \epsilon_{r}$, where $\epsilon_{r}$ is the $\alpha$-p resonance energy. In this case, where the $2 p$-wave function keeps the pure $\left(p_{3 / 2}\right)^{2}$ configuration, it was confirmed that the decay process is well described as a sequential $2 p$ emission [33].

Taking PCE into account, the resonance energy is decreased mainly due to the attractive pairing interaction. Figure 1 schematically describes this situation. In Ref. [33], the finiterange, density-independent Minnesota potential was employed to describe the pairing force [44], and then the strongly correlated $2 p$ emission was suggested. ${ }^{1}$ For the spatial correlation in this process to occur, a mixture of other configurations from $\left(p_{3 / 2}\right)^{2}$ plays an essential role $[29,36]$. Also, especially with the light core nucleus, the induced correlation by the recoil

\footnotetext{
${ }^{1}$ We found a typo in Table I of Ref. [33]: " $S=0(1)$ " should be corrected as " $S=1(0)$ ".
} 


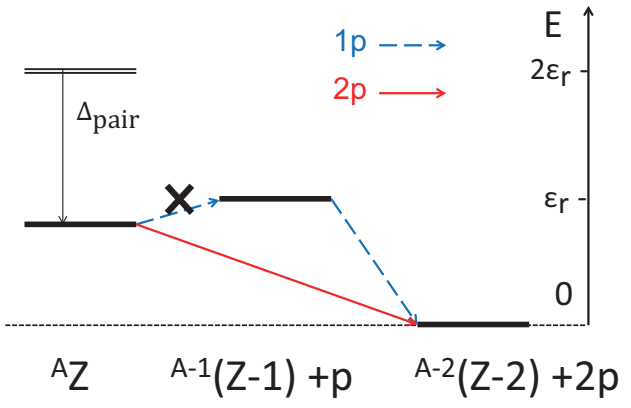

FIG. 1. Schematic figure of level scheme, in which the correlated $2 p$ emission becomes dominant.

term, $\left(\boldsymbol{p}_{1} \cdot \boldsymbol{p}_{2}\right) / A_{\mathrm{c}} m$, can be noticeable. We check its effect on the $Q$ value in the next section.

\section{NUMERICAL CALCULATION}

\section{A. Initial state}

In order to fix the initial state for time evolution, we employ the same confining potential, $V_{\mathrm{c}-\mathrm{p}}^{\mathrm{conf}}(r)$, as in Ref. [33]. This confining potential method has provided good approximations for quantum resonance phenomena [23,45-47], together with an intuitive way to understand their dynamics. The initial state solved within the confining Hamiltonian can be expanded on the eigenbasis of the original Hamiltonian: $|\Psi(0)\rangle=$ $\sum_{N} F_{N}(0)\left|E_{N}\right\rangle$. Thus, the time evolution is represented as

$$
|\Psi(t)\rangle \equiv \exp \left[-i t \frac{H_{3 \mathrm{~b}}}{\hbar}\right]|\Psi(0)\rangle=\sum_{N} F_{N}(t)\left|E_{N}\right\rangle
$$

where $F_{N}(t)=e^{-i t E_{N} / \hbar} F_{N}(0)$. It is worthwhile to note that the time-invariant discrete energy spectrum can be given as

$$
d\left(E_{N}\right)=\left|F_{N}(0)\right|^{2}=\left|F_{N}(t)\right|^{2} .
$$

If one takes the continuous energy limit, $d(E)$ resembles the Breit-Wigner spectrum, which characterizes the quantum resonance properties of concerning radioactive process [48].

In Fig. 2, we plotted the normalized density distribution for the initial state. That is,

$$
\rho\left(t ; r_{1}, r_{2}, \theta_{12}\right)=8 \pi^{2} r_{1}^{2} r_{2}^{2} \sin \theta_{12}\left|\Psi\left(t ; r_{1}, r_{2}, \theta_{12}\right)\right|^{2},
$$

at $c t=0 \mathrm{fm}$. For convenience, $\rho$ is translated to a function of the relative distance between the two protons, $r_{\mathrm{p}-\mathrm{p}}=\left(r_{1}^{2}+\right.$ $\left.r_{2}^{2}-2 r_{1} r_{2} \cos \theta_{12}\right)^{1 / 2}$, and that between the core and the center of mass of two protons, $r_{\mathrm{c}-\mathrm{pp}}=\left(r_{1}^{2}+r_{2}^{2}+2 r_{1} r_{2} \cos \theta_{12}\right)^{1 / 2} / 2$. From Fig. 2, we find a similar result in Ref. [33], where a finite-range Minnesota pairing was used instead: the higher peak at $r_{\mathrm{p}-\mathrm{p}} \simeq 2.0 \mathrm{fm}$ and $r_{\mathrm{c}-\mathrm{pp}} \simeq 2.5 \mathrm{fm}$, as well as at $\theta_{12} \simeq \pi / 6$, indicates a strong localization of two protons. The similar discussion can be found in, e.g., Refs. [29,49], where the pairing correlation as well as the Pauli principle play a fundamental role. Notice also that this localization is attributable to the spin-singlet configuration, suggesting a diproton correlation [37].
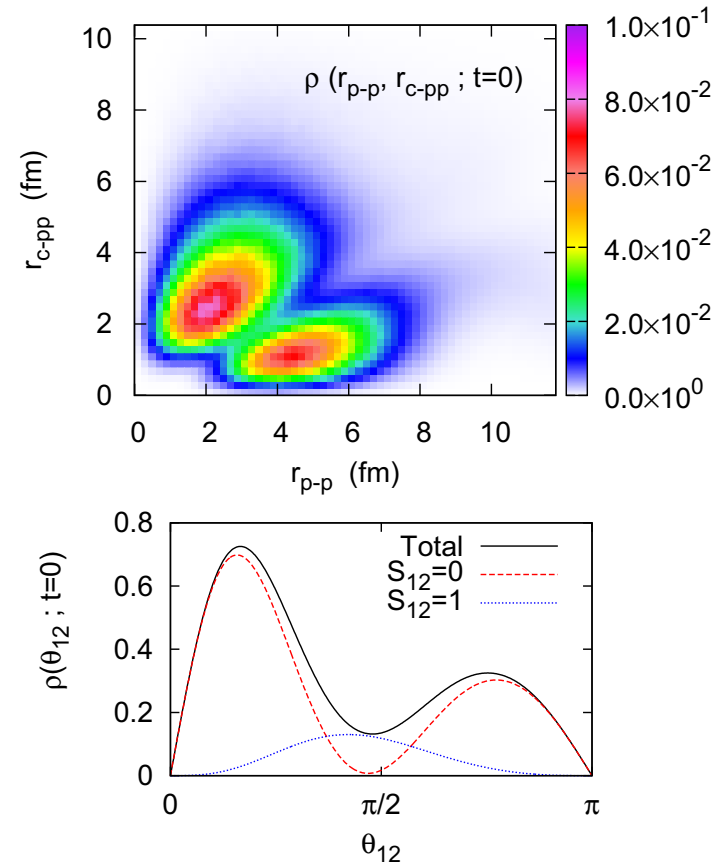

FIG. 2. The density distribution for the initial $2 p$ state obtained with the surface SDDC pairing interaction. (i) Top panel: the distribution as a function of $r_{\mathrm{p}-\mathrm{p}}$ and $r_{\mathrm{c}-\mathrm{pp}}$. (ii) Bottom panel: the angular distribution as a function of the opening angle, $\theta_{12}$, between two protons. This is obtained by integrating $\rho\left(r_{1}, r_{2}, \theta_{12}\right)$ for the radial coordinates, $r_{1}$ and $r_{2}$. The spin singlet and spin-triplet components are also plotted.

For this initial state, the $Q$ value is obtained as

$$
Q_{2 \mathrm{p}}=\left\langle\Psi(0)\left|H_{3 \mathrm{~b}}\right| \Psi(0)\right\rangle=1.37 \mathrm{MeV}
$$

with our surface SDDC pairing interaction. This includes the negative PCE, where not only the pairing interaction but also the induced correlation from the recoil term give finite values. That is,

$$
\Delta_{\text {pair }}=\left\langle\frac{\boldsymbol{p}_{1} \cdot \boldsymbol{p}_{2}}{A_{\mathrm{c}} m}\right\rangle+\left\langle v_{\mathrm{p}-\mathrm{p}}\right\rangle=-6.28 \mathrm{MeV},
$$

where $\left\langle\left(\boldsymbol{p}_{1} \cdot \boldsymbol{p}_{2}\right) / A_{\mathrm{c}} m\right\rangle=-1.46 \mathrm{MeV}$ and $\left\langle v_{\mathrm{p}-\mathrm{p}}\right\rangle=-4.82$ $\mathrm{MeV}$. Obviously, the pairing interaction makes a major contribution in reproducing the empirical $Q$ value. In our case, the recoil term effect is also noticeable, which exhausts about $25 \%$ of total PCE. This feature of the center-of-mass effect may take place when the masses of ingredient particles are comparable.

\section{B. Time evolution}

In Fig. 3, we plotted the time development of $2 p$ state, in terms of the probability-density distribution. It is well described that the confined two protons at $c t=0$ are released during the time development. The probability density outside the core-proton barrier gradually increases, indicating an evacuation of two protons. In order to monitor their decay dynamics more preciously, it is helpful to focus on the 

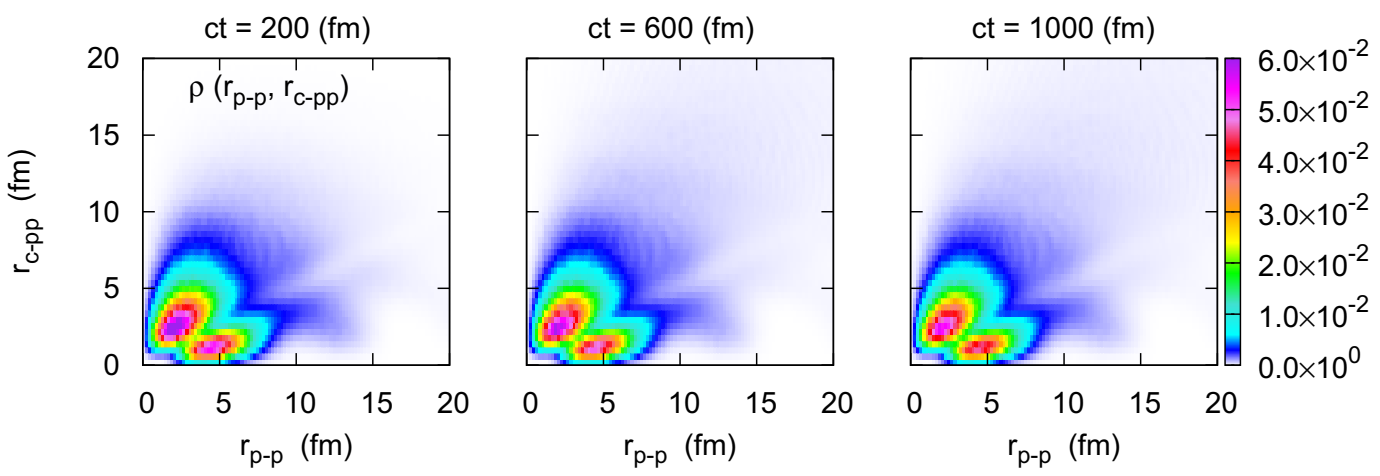

FIG. 3. Time-dependent $2 p$-density distribution plotted as a function of $r_{\mathrm{p}-\mathrm{p}}$ and $r_{\mathrm{c} \text {-pp }}$. The surface SDDC pairing interaction is employed.

projected decay state [27]. That is,

$$
\left|\Psi_{d}(t)\right\rangle \equiv|\Psi(t)\rangle-\beta(t)|\Psi(0)\rangle,
$$

with $\beta(t)=\langle\Psi(0) \mid \Psi(t)\rangle$. Because the initial state is well confined, this projected decay state mainly represents the outgoing components released from around the core. In Fig. 4, we plot the density distribution of the projected decay state normalized at each point of time. That is,

$$
\rho_{d}\left(t ; r_{1}, r_{2}, \theta_{12}\right)=\frac{8 \pi^{2} r_{1}^{2} r_{2}^{2} \sin \theta_{12}\left|\Psi_{d}\left(t ; r_{1}, r_{2}, \theta_{12}\right)\right|^{2}}{N_{d}(t)},
$$

where $N_{d}(t)=\left\langle\Psi_{d}(t) \mid \Psi_{d}(t)\right\rangle=1-|\beta(t)|^{2}$ is the decay probability. In Fig. 4 , the strongly correlated $2 p$ emission is suggested with our surface SDDC pairing model. The diproton correlation, which can be detected as a peak at $r_{\mathrm{p}-\mathrm{p}} \simeq 5 \mathrm{fm}$ and $r_{\mathrm{c}-\mathrm{pp}} \simeq 10 \mathrm{fm}$, as well as at $\theta_{12} \simeq \pi / 6$, is dominant during the time evolution. Notice also that the sequential $2 p$ emission, which is graphically indicated as a ridge along the $r_{\mathrm{c}-\mathrm{pp}} \simeq r_{\mathrm{p}-\mathrm{p}} / 2$ line [33], is strongly suppressed. This dynamical behavior of protons is similar to that suggested from the finite-range Minnesota pairing model [33].

\section{Decay width}

We next investigate the decay width, which is one of the directly measurable quantities of the $2 p$ emission. From the decay probability $N_{d}(t)$, the $2 p$-decay width is calculated as

$$
\Gamma(t)=-\hbar \frac{d}{d t} \ln \left[1-N_{d}(t)\right]=\frac{\hbar}{1-N_{d}(t)} \frac{d}{d t} N_{d}(t),
$$

where $1-N_{d}(t)$ indicates the survival probability. It is worthwhile to note that, if the time evolution follows the exponential decay rule, which is a fundamental property of radioactive processes, the decay probability is given as $N_{d}(t)=1-\exp \left(-t \Gamma_{c} / \hbar\right)$. Here $\Gamma_{c}$ is the constant decay width. In this case, $\Gamma(t)$ becomes identical to $\Gamma_{c}$, which determines the mean lifetime of this system, $\tau=\hbar / \Gamma_{c}$.
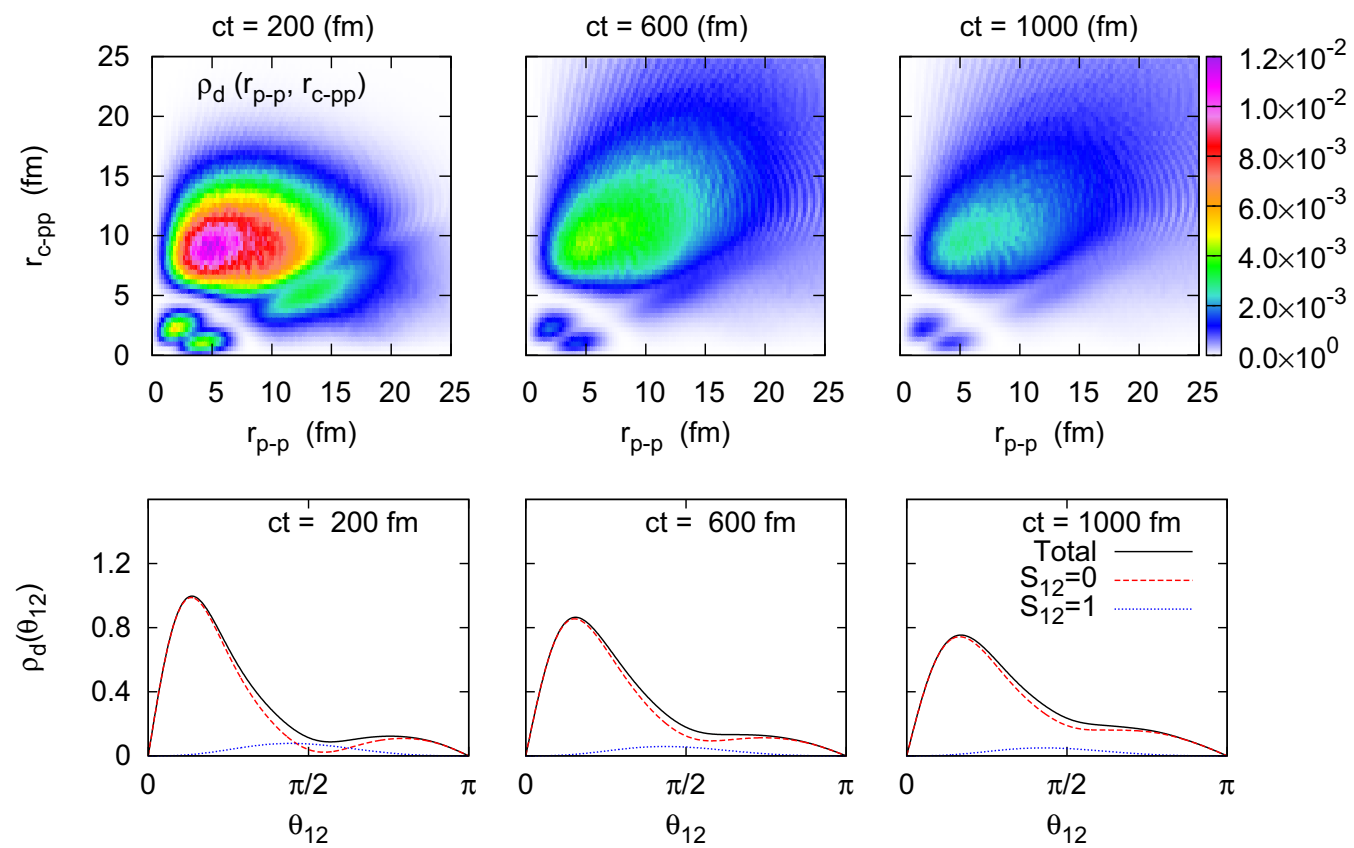

FIG. 4. Time-dependent $2 p$-density distribution of the decay state $\rho_{d}(t)$, given by Eq. (16). The surface SDDC pairing interaction is employed. These are plotted in the same manner as in Fig. 2. 
TABLE I. Parameters for the SDDC pairing interaction used in this article. $E_{\text {cut }}=40 \mathrm{MeV}$. Resultant $Q$ values of the $2 p$ emission of ${ }^{6} \mathrm{Be}$ and the corresponding total, spin-singlet, and spin-triplet decay widths at $c t=1000 \mathrm{fm}$ are also present. Same results but with the softened, finite-range Minnesota pairing model are obtained from Ref. [33].

\begin{tabular}{lcccccc}
\hline \hline & $\begin{array}{c}w_{0} \\
\left(\mathrm{MeV} \mathrm{fm}^{3}\right)\end{array}$ & $\eta$ & $\begin{array}{c}Q_{2 \mathrm{p}} \\
(\mathrm{keV})\end{array}$ & $\begin{array}{c}\Gamma \\
(\mathrm{keV})\end{array}$ & $\begin{array}{c}\Gamma_{S=0} \\
(\mathrm{keV})\end{array}$ & $\begin{array}{c}\Gamma_{S=1} \\
(\mathrm{keV})\end{array}$ \\
\hline SDDC (this work) & -767.398 & 1.04 & 1370.7 & 34.7 & 33.4 & 1.3 \\
Minnesota [33] & & & 1370.7 & 88.2 & 87.1 & 1.1 \\
Experiment [38] & & $1371 \pm 5$ & $92 \pm 6$ & & \\
\hline \hline
\end{tabular}

In the following, for a comparison with our SDDC model, we fetch the same result with a softened finite-range Minnesota pairing model [33]. Notice that, as presented in Table I, both pairing models are adjusted so as to reproduce the same $Q$ value. This is an important constraint because, for radioactive processes governed by the quantum tunneling effect, even a small difference in the $Q$ value can lead to a large change of the decay width [21-25,50]. However, as an intuitive shortcoming in Ref. [33], we should also warn that fitting Minnesota potential to the $Q$ value leads to the inconsistency in the experimental scattering length. Also, the core-proton potential and the cutoff parameters are common in both cases.

In Fig. 5, we plot the survival probability and decay width as functions of time. After a sufficient time evolution, we finally

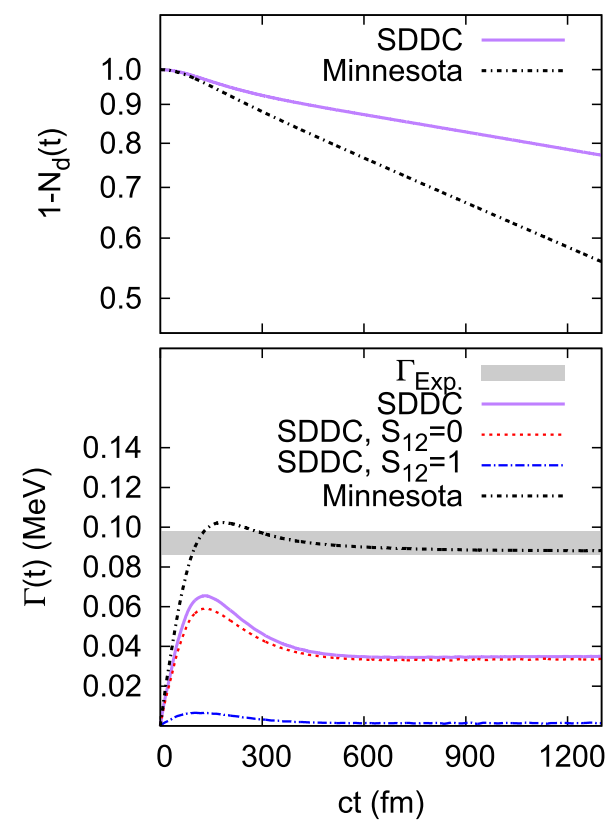

FIG. 5. (i) Top panel: survival probability, $1-N_{d}(t)$, obtained with the surface SDDC pairing interaction. For a comparison, the same result but with the finite-range Minnesota pairing is taken from Ref. [33]. These are plotted in logarithmic scale. (ii) Bottom panel: $2 p$-decay width of ${ }^{6} \mathrm{Be}$ calculated with the surface SDDC and the finite-range Minnesota pairing interactions. In SDDC case, the spinsinglet $\left(S_{12}=0\right)$ and spin-triplet $\left(S_{12}=1\right)$ widths are both plotted. Experimental result, $\Gamma_{2 p}=92 \pm 6 \mathrm{keV}$, is indicated by the shaded area [38]. obtain the exponential decay rule and thus the convergence of decay width. From the Krylov-Fock theorem [48,51], this exponential decay coincides with that of the energy distribution $d\left(E_{N}\right)$, and approximates the Breit-Wigner spectrum.

For the deviation from exponential decay rule in the long-time scale, there have been several statements of its existence in radioactive processes [52-56]. Investigation of this long-time deviation is, however, not feasible with present time-dependent model, because the reflected wave at $R_{\mathrm{box}}$ invokes an unphysical deviation. In order to disinfect this "contamination" by the unphysically reflected wave, one needs to employ, e.g., the absorption boundary condition [57,58]. Because this improvement is technically demanding, we leave it for future work. We emphasize that our conclusion based on the resultant decay width is independent of this reflected contamination.

In Table I, the $\Gamma(t)$ value at $c t=1000 \mathrm{fm}$ is tabulated. In our result, the SDDC pairing interaction underestimates the experimental $2 p$-decay width, whereas the Minnesota pairing showed a good agreement with it. In Fig. 5, the partial decay widths for the spin-singlet and spin-triplet channels are also plotted [33]: $\Gamma(t)=\Gamma_{S=0}(t)+\Gamma_{S=1}(t)$. One finds again the dominance of the spin-singlet configuration in $2 p$ emission consistently to the density distribution in Fig. 4 . The exact values of $\Gamma_{S=0,1}(t)$ at $c t=1000 \mathrm{fm}$ are also summarized in Table I. With the SDDC pairing, the spin-singlet $2 p$-decay width is remarkably small compared with the Minnesota pairing case, whereas the spin-triplet width shows the similar values. Because of the same setting except the pairing models in two cases, the different $2 p$-decay widths should be purely attributed to the pairing properties.

Figure 6 displays the discrete energy spectra $d\left(E_{N}\right)$ and their continuous distributions smeared by a Cauchy-Lorentz function. The spectrum width obtained with the SDDC pairing model is narrower than that of the Minnesota pairing model. This result coincides with the converged $\Gamma$ values.

Last, we confirmed that our conclusion does not change even if we employ a smaller value of $E_{\text {cut }}$ : we calculated the decay width with $E_{\text {cut }}=32 \mathrm{MeV}$, with the SDDC pairing

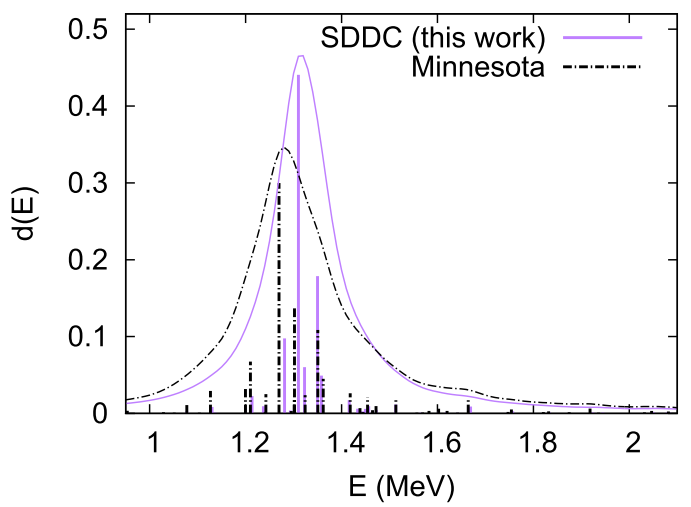

FIG. 6. Time-invariant discrete energy distribution $d\left(E_{N}\right)$ obtained with the surface SDDC pairing interaction. Its continuous distribution, plotted in an arbitrary scale, is obtained by smearing $d\left(E_{N}\right)$ with a Cauchy-Lorentz function, whose full width at the half maximum is $0.1 \mathrm{MeV}$. The same plot for the Minnesota pairing case is also displayed. 
TABLE II. Parameters for SDDC pairing interactions used in Sec. IIID (upper three rows) and Sec. IIIE (middle two rows). Resultant $Q_{2 \mathrm{p}}$ and $\Gamma$ are displayed in the same manner as Table I.

\begin{tabular}{lcccccc}
\hline \hline & $\begin{array}{c}w_{0} \\
\left(\mathrm{MeV} \mathrm{fm}^{3}\right)\end{array}$ & $\begin{array}{c}R_{f} \\
(\mathrm{fm})\end{array}$ & $\begin{array}{c}a_{f} \\
(\mathrm{fm})\end{array}$ & $\eta$ & $\begin{array}{c}Q_{2 \mathrm{p}} \\
(\mathrm{keV})\end{array}$ & $\begin{array}{c}\Gamma \\
(\mathrm{keV})\end{array}$ \\
\hline $\begin{array}{l}\text { Default SDDC } \\
\text { Steep }\end{array}$ & -767.398 & 1.68 & 0.615 & 1.04 & 1370.7 & 34.7 \\
Smooth & $(\mathrm{same})$ & 0.84 & $(\mathrm{same})$ & 2.53 & 1370.8 & 33.3 \\
Volume & -525.5 & 1.68 & 0.615 & 0 & 1370.6 & 19.7 \\
Emitter & -1036.8 & $(\mathrm{same})$ & (same) & 1.80 & 1367.6 & 90.3 \\
Experiment [38] & & & & & $1371 \pm 5$ & $92 \pm 6$ \\
\hline \hline
\end{tabular}

interaction refitted to reproduce the empirical scattering length and the $Q$ value, $Q_{2 \mathrm{p}}=1.37 \mathrm{MeV}$. Then we obtained the same decay-width value as in the original energy cutoff case in Table I.

\section{Density dependence}

In this part, we discuss in detail the inconsistency of $\Gamma_{2 \mathrm{p}}$ and $Q_{2 p}$. First we should remember that the asymptotic pairing strength $w_{0}$ has been adjusted consistently to the vacuum scattering length $a_{0}$, which gives the first constraint from experimental results. For the other two observables, $Q_{2 \mathrm{p}}$ and $\Gamma_{2 p}$ to be reproduced simultaneously, we can manipulate the density dependence $w(r)$, only around the core nucleus.

For this purpose, in addition to our default SDDC parameters, we test two sets of parameters, steep and smooth, as summarized in Table II. Visual plots of these $w(r)$ are shown in Fig. 7. In these cases, we modify the radial parameter, $R_{f}$ in Eq. (4), from the default value. Then, we readjust the parameter $\eta$ to reproduce the empirical $Q$ value. Consequently, in the steep SDDC case, the density-dependent strength should be positive deeply inside the core, meaning that the $2 p$ interaction should be repulsive there due to our $Q$-value fitting purpose. On the other hand, in the smooth SDDC case, $w(r)$ is always attractive with a smooth change around the core-proton barrier. We remind that the asymptotic value $w_{0}$ is common in all the cases. Note also that we change these parameters only in the pairing interaction, whereas the core-proton interaction $V_{\mathrm{WS}}(r)$ has been common in all the cases. Namely, resonance parameters of $\alpha-p$ remain unchanged.

In Fig. 7, our resultant $\Gamma(t)$ are present: there is actually no significant difference in the three cases. Namely, the density dependence of pairing strength plays a minor role in the $2 p$ penetrability, whereas only the asymptotic strength can control it. It also means that there has been no way to resolve the trinity problem of $Q_{2 \mathrm{p}}, \Gamma_{2 \mathrm{p}}$, and $a_{0}$, as long as with the simple SDDC pairing model. Indeed, this impossible trinity was found also in Ref. [33], where the softened Minnesota model should affect the consistency to the experimental scattering length.

\section{E. Sensitivity to asymptotic interaction}

In order to investigate the effect of the asymptotic interaction, we repeat the same calculation but changing the $w_{0}$ values in the following. Although it leads to an inconsistency to the empirical scattering length, we expect to obtain a hint
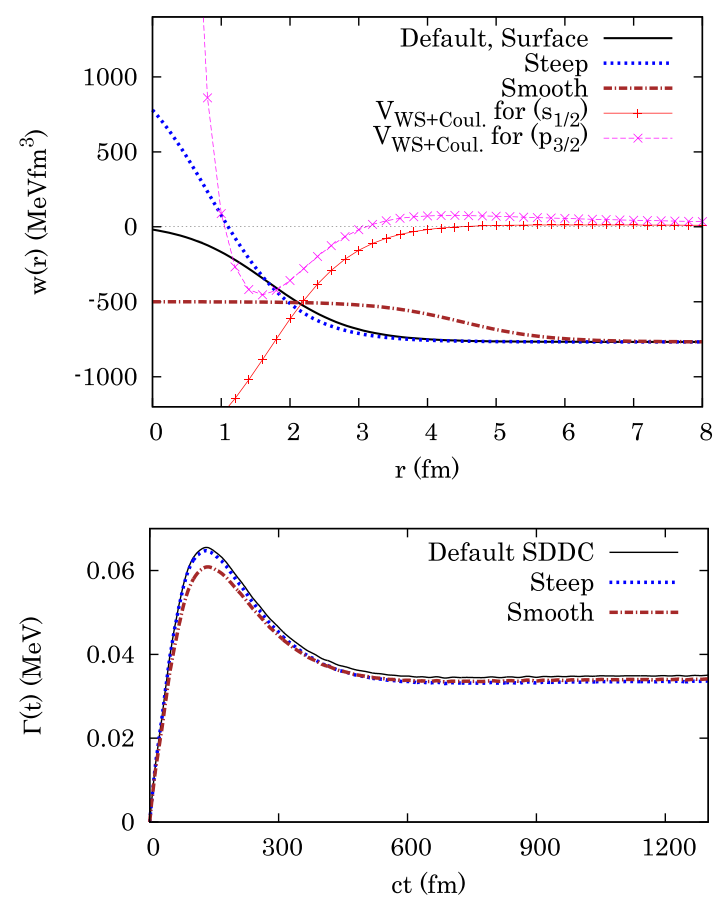

FIG. 7. (i) Top panel: density dependence of the SDDC pairing interaction $w(r)$ in the default (surface), steep, and smooth cases. The core-proton potential is also plotted in the arbitrary scale. (ii) Bottom panel: corresponding result of $2 p$-decay width.

for further sophistication of the theoretical model. Those sets of parameters are displayed in Table II, named volume and emitter SDDC interactions. In the volume SDDC case, we fix $\eta=0$, and fit $w_{0}$ to the empirical $Q$ value. Thus, the pairing strength becomes independent of the radial density. This interaction imitates the so-called volume type of the pairing energy functional in DFT calculations [7-9]. In the emitter SDDC case, on the other hand, we search an adequate set of $\left(\eta, w_{0}\right)$, which can reproduce the empirical $Q_{2 \mathrm{p}}$ and $\Gamma_{2 \mathrm{p}}$ simultaneously. Consequently, $\eta=1.8$ and $w_{0}=$ $-1036.8 \mathrm{MeV} \mathrm{fm}^{3}$ are obtained.

In the top panel of Fig. 8, we plot the contact pairing strength for these SDDC parametrizations. It is worthwhile to mention that, with the emitter SDDC model, due to its deeper $w_{0}$ value, two protons in vacuum have a larger correlation energy.

Figure 9 shows the decay width obtained with different asymptotic strengths. Obviously, one can find that the stronger pairing in the asymptotic region yields the larger decay width. This is consistent with other theoretical results [19-25]. It is also remarkable that this asymptotic-pairing sensitivity can be concluded even in the equivalent kinematic condition, which has been realized with the standard $Q$ value in our calculations.

The asymptotic sensitivity may be found with other kinds of the pairing model. In Appendix, we show another example with the Minnesota pairing model, which is not density dependent and has a finite range. In that section, by tuning the range and strength parameters of the Minnesota pairing, the sensitivity of the $2 p$-decay width is confirmed.

Our time-dependent model can provide an intuitive way to study the asymptotic-pairing sensitivity of $2 p$ dynamics. For 


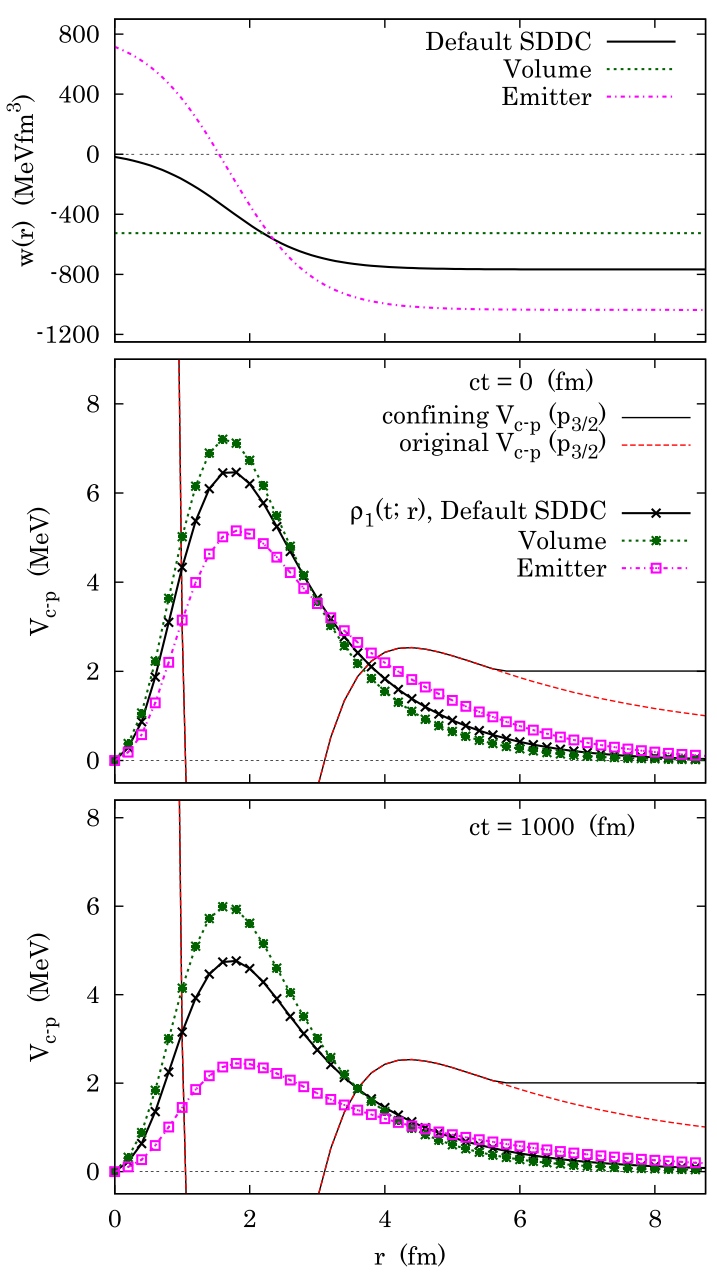

FIG. 8. (i) Top panel: The radial strength $w(r)$ for three SDDC pairing potentials, $v_{\mathrm{p}-\mathrm{p}}^{(N)}\left(\boldsymbol{r}_{1}, \boldsymbol{r}_{2}\right)=w\left(r_{1}\right) \cdot \delta\left(\boldsymbol{r}_{1}-\boldsymbol{r}_{2}\right)$. (ii) Middle and bottom panels: The one-proton density-distribution at $c t=0$ and $1000 \mathrm{fm}$, respectively. These distributions are plotted in an arbitrary scale. Confining and original potentials in the $\left(p_{3 / 2}\right)$ channel of $\alpha-p$ subsystem are also plotted.

this purpose, in the lower two panels in Fig. 8, we present the one-proton probability-density distribution of the initial and

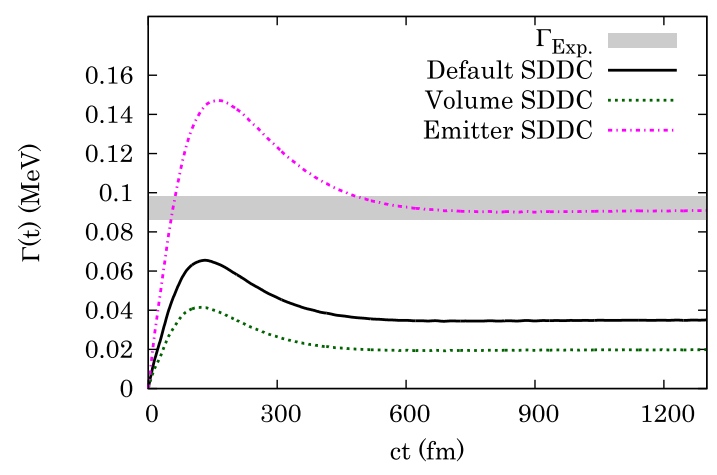

FIG. 9. $2 p$-decay width of ${ }^{6} \mathrm{Be}$ obtained with the default, volume, and emitter SDDC pairing models. time-developed states. That is,

$$
\rho_{1}(t ; r)=8 \pi^{2} \int_{0}^{R_{\mathrm{box}}} d r_{2} r_{2}^{2} \int_{-1}^{1} d\left(\cos \theta_{12}\right)\left|\Psi\left(t ; r, r_{2}, \theta_{12}\right)\right|^{2} .
$$

Because our $2 p$-basis functions are antisymmetrized, $\left|\Psi\left(\boldsymbol{r}_{1}, \boldsymbol{r}_{2}\right)\right|^{2}$ is symmetric for the exchange of $\boldsymbol{r}_{1}$ and $\boldsymbol{r}_{2}$. Thus, $\rho_{1}(t ; r)$ represents the mean radial distribution of $2 p$ probability. In these panels, with the default (surface) or emitter SDDC, the probability density shows a dispersed shape. This is of course a product of the strong $p$ - $p$ attraction in vacuum: the two-proton subsystem more strongly favors the outside from the potential barrier. This effect then yields a looser stability corresponding to the larger decay width. On the other hand, in the volume SDDC case, the two-proton density hardly diverges with the isotropically attractive pairing.

In order to resolve the impossible trinity problem in Sec. III D, now the qualitative suggestion appears: the pairing model should satisfy both (i) the consistency with the asymptotic scattering problem, and (ii) the dynamical effect on two protons for tunneling, as seen in Fig. 8. Possible ways in practice include a nontrivial parametrization of the density dependence [59], and/or the phenomenological three-body force $[17,19,60]$. Note also that, in our present model, this dynamical process is driven by the total Hamiltonian, which is not time dependent nor self-consistent to the $2 p$ state. This assumption will need to be addressed in forthcoming studies.

\section{SUMMARY}

We have discussed the dependence of $2 p$ radioactivity on nuclear pairing models within the time-dependent three-body model calculations. Comparing the zero-range SDDC and the finite-range Minnesota pairing forces, the $2 p$ dynamics is interpreted as the correlated $2 p$ emission similarly in both cases.

Evaluating the absolute decay probabilities, we found that the two-proton decay width is sensitive to the pairing model in usage. Utilizing the SDDC parametrizations, we showed that the asymptotic strength of the pairing interaction essentially controls the $2 p$-decay width. This sensitivity exists even if we exclude the kinematic effect by reproducing the equivalent condition on the emitted $Q$ value. On the other hand, the density-dependence effect around the core plays a minor role in this field.

With the simple SDDC pairing model, there remains an impossible trinity problem of $Q_{2 \mathrm{p}}, \Gamma$, and the two-nucleon scattering length in vacuum, $a_{0}$. In order to reproduce whole of these two-body and three-body properties consistently to the experiments, further model sophistication is necessary. One possible approach is to employ a nontrivial parametrization of the density dependence for the pairing interaction [59], and another is the phenomenological three-body force $[17,19,60]$. Because these considerable solutions inevitably harm the simplicity of the present model, we leave these developments for future study.

Comparison with other kinds of experimental data, e.g., momentum distributions in Refs. [17,19,61], is also an 
important task for future work. For this purpose, however, the present model space should be expanded sufficiently to handle the long-range Coulomb effects. Although the computational cost is highly increased, it may provide another procedure to validate the pairing models.

Another direction of progress may be the implementation of our idea to the mean-field calculations [62-65]. Because our three-body Hamiltonian itself is not time dependent nor self-consistent, it is not completely clear whether similar pairing sensitivity exists in the SCMF or DFT calculations. Implementing our procedure to this framework enables us to perform the systematic investigation along the $2 p$-drip line, utilizing the $2 p$-decay data as the reference quantities. A wide experimental survey for the $2 p$-emitter candidates could be profitable for this purpose [14].

\section{ACKNOWLEDGMENTS}

T.O. sincerely thanks L. Fortunato, A. Vitturi, K. Hagino, and H. Sagawa for fruitful discussions. This work was supported by the Academy of Finland and University of Jyvaskyla within the FIDIPRO program and within the Centre of Excellence Programme 2012-2017 (Nuclear and Accelerator Based Programme at JYFL). T.O. acknowledges the financial support from the PRAT 2015 project IN:Theory at the University of Padova (Project Code CPDA154713). We acknowledge the CSC-IT Center for Science Ltd., Finland, for the allocation of computational resources.

\section{APPENDIX: CONTACT AND MINNESOTA PAIRING MODELS}

In this Appendix, we discuss a connection of the zero-range pairing model to the Minnesota type, which was employed in Ref. [33], with several results for the $2 p$-decay width. The $p-p$ Minnesota potential used in Ref. [33] was given as

$$
\begin{aligned}
v_{\mathrm{p}-\mathrm{p}}^{(N)}=V_{\mathrm{Min}}= & v_{r} e^{-d^{2} / 2 q^{2}}+v_{s} e^{-d^{2} / 2 \kappa_{s}^{2} q^{2}} \hat{P}_{S=0} \\
& +v_{t} e^{-d^{2} / 2 \kappa_{t}^{2} q^{2}} \hat{P}_{S=1},
\end{aligned}
$$

where $d \equiv\left|\boldsymbol{r}_{1}-\boldsymbol{r}_{2}\right|, \quad v_{r}=156 \mathrm{MeV}, v_{s}=-91.85 \mathrm{MeV}$, $v_{t}=-178 \mathrm{MeV}, q=0.5799 \mathrm{fm}, \kappa_{s}=1.788$, and $\kappa_{t}=1.525$. $\hat{P}_{S=0(1)}$ is the projection to the spin-singlet (triplet) channel. Remember that $v_{r}$ was softened from the original value, $v_{r}=200 \mathrm{MeV}$ [44], in order to reproduce the reference $Q$ value, $Q_{2 \mathrm{p}}=1.37 \mathrm{MeV}$. Here the first term describes a soft repulsive core.

Decomposing $d^{2}=x^{2}+y^{2}+z^{2}$, the first term in Eq. (A1) reads

$$
v_{r} e^{-d^{2} / 2 q^{2}}=w_{r} \frac{e^{-x^{2} / 2 q^{2}} e^{-y^{2} / 2 q^{2}} e^{-z^{2} / 2 q^{2}}}{(2 \pi)^{3 / 2} q^{3}},
$$

where $w_{r}=v_{r}(2 \pi)^{3 / 2} q^{3}=479.1 \mathrm{MeV} \mathrm{fm}^{3}$, and similarly as expected for the following attraction terms. Utilizing a well

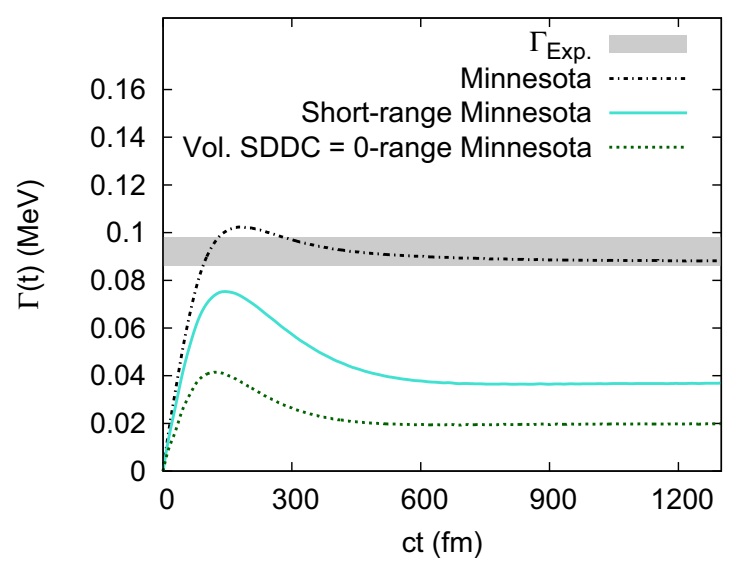

FIG. 10. Time-dependent $2 p$-decay width of ${ }^{6} \mathrm{Be}$ obtained with several Minnesota-pairing forces.

known formula,

$$
\lim _{q \rightarrow 0} \frac{e^{-x^{2} / 2 \kappa^{2} q^{2}}}{q \sqrt{2 \pi}}=\delta(x / \kappa)=|\kappa| \delta(x),
$$

then at zero-range limit, we obtain

$$
\lim _{q \rightarrow 0} V_{\operatorname{Min}}\left(\boldsymbol{r}_{1}, \boldsymbol{r}_{2}\right)=w_{0} \delta\left(\boldsymbol{r}_{1}-\boldsymbol{r}_{2}\right)
$$

where $w_{0}=w_{r}+\kappa_{s}^{3} w_{s}=-1133.4 \mathrm{MeV} \mathrm{fm}^{3}$. Indeed, this zero-range form is identical to the volume type of the pairing force used in Sec. III E. Notice also that, for the two-proton basis in $0^{+}$configuration, matrix elements of the spin-triplet contact potential become zero automatically from the angularmomentum algebra [66].

Employing the volume contact pairing given in Eq. (A4), however, we confirmed that the $\alpha+p+p$ three-body system fictionally becomes bound with $Q_{2 p} \simeq-1.3 \mathrm{MeV}$. In order to reproduce the experimental $Q$ value, we need to use the shallower strength as in Table II in the main text. Then, we obtain $\Gamma=88.2$ and $19.7 \mathrm{keV}$ with the finite-range and zero-range Minnesota potentials, respectively. To reinforce our result, we repeat the same calculation but with the shorter range, $q / \sqrt{2} \simeq 0.41 \mathrm{fm}$ in Eq. (A1). In this case, we need to employ the enhancement factor, $f=2.047$, to reproduce the reference $Q$ value: $v_{\mathrm{p}-\mathrm{p}}^{(N)}=f \cdot V_{\operatorname{Min}}(q / \sqrt{2})$.

In Fig. 10, all the resultant $2 p$-decay widths are displayed. As expected, the short-range Minnesota case yields the medium value of the decay width between the default and zero-range Minnesota cases. Because Minnesota forces are density independent, this sensitivity of $2 p$-decay width is purely attributable to the asymptotic scattering property, which is governed by the choice of parameters.
[1] D. Brink and R. Broglia, Nuclear Superfluidity: Pairing in Finite Systems, Cambridge Monographs on Particle Physics,
Nuclear Physics and Cosmology (Cambridge University Press, Cambridge, UK, 2005). 
[2] Fifty Years of Nuclear BCS: Pairing in Finite Systems, edited by R. A. Broglia and V. Zelevinsky (World Scientific, Singapore, 2013).

[3] D. J. Dean and M. Hjorth-Jensen, Rev. Mod. Phys. 75, 607 (2003).

[4] M. Bender, P.-H. Heenen, and P.-G. Reinhard, Rev. Mod. Phys. 75, 121 (2003).

[5] J. Dobaczewski, W. Nazarewicz, T. R. Werner, J. F. Berger, C. R. Chinn, and J. Dechargé, Phys. Rev. C 53, 2809 (1996).

[6] T. Lesinski, T. Duguet, K. Bennaceur, and J. Meyer, Eur. Phys. J. A 40, 121 (2009); (private communications).

[7] N. Sandulescu, P. Schuck, and X. Viñas, Phys. Rev. C 71, 054303 (2005).

[8] A. Pastore, F. Barranco, R. A. Broglia, and E. Vigezzi, Phys. Rev. C 78, 024315 (2008).

[9] A. Pastore, J. Margueron, P. Schuck, and X. Viñas, Phys. Rev. C 88, 034314 (2013).

[10] M. Grasso, Phys. Rev. C 87, 064308 (2013).

[11] S. A. Changizi and C. Qi, Phys. Rev. C 91, 024305 (2015).

[12] N. Pillet, N. Sandulescu, P. Schuck, and J.-F. Berger, Phys. Rev. C 81, 034307 (2010).

[13] J. Erler, N. Birge, M. Kortelainen, W. Nazarewicz, E. Olsen, A. M. Perhac, and M. Stoitsov, Nature (London) 486, 509 (2012).

[14] E. Olsen, M. Pfützner, N. Birge, M. Brown, W. Nazarewicz, and A. Perhac, Phys. Rev. Lett. 110, 222501 (2013).

[15] V. I. Goldansky, Nucl. Phys. 19, 482 (1960).

[16] V. I. Goldansky, Nucl. Phys. 27, 648 (1961).

[17] L. Grigorenko, T. Wiser, K. Miernik, R. Charity, M. Pftzner, A. Banu, C. Bingham, M. Ćwiok, I. Darby, W. Dominik, J. Elson, T. Ginter, R. Grzywacz, Z. Janas, M. Karny, A. Korgul, S. Liddick, K. Mercurio, M. Rajabali, K. Rykaczewski et al., Phys. Lett. B 677, 30 (2009).

[18] B. Blank and M. Pĺoszajczak, Rep. Prog. Phys. 71, 046301 (2008).

[19] L. V. Grigorenko, Phys. Part. Nucl. 40, 674 (2009).

[20] M. Pfützner, M. Karny, L. V. Grigorenko, and K. Riisager, Rev. Mod. Phys. 84, 567 (2012).

[21] L. V. Grigorenko, R. C. Johnson, I. G. Mukha, I. J. Thompson, and M. V. Zhukov, Phys. Rev. Lett. 85, 22 (2000).

[22] L. V. Grigorenko and M. V. Zhukov, Phys. Rev. C 76, 014008 (2007).

[23] T. Maruyama, T. Oishi, K. Hagino, and H. Sagawa, Phys. Rev. C 86, 044301 (2012).

[24] D. S. Delion, R. J. Liotta, and R. Wyss, Phys. Rev. C 87, 034328 (2013).

[25] R. Lundmark, C. Forssén, and J. Rotureau, Phys. Rev. A 91, 041601 (2015).

[26] K. Hagino and H. Sagawa, Phys. Rev. C 89, 014331 (2014).

[27] C. Bertulani, M. Hussein, and G. Verde, Phys. Lett. B 666, 86 (2008).

[28] J. Margueron, H. Sagawa, and K. Hagino, Phys. Rev. C 76, 064316 (2007).

[29] K. Hagino, H. Sagawa, J. Carbonell, and P. Schuck, Phys. Rev. Lett. 99, 022506 (2007).

[30] V. Efimov, Phys. Lett. B 33, 563 (1970).

[31] P. F. Bedaque, H.-W. Hammer, and U. van Kolck, Phys. Rev. Lett. 82, 463 (1999).

[32] E. Hiyama and M. Kamimura, Phys. Rev. A 90, 052514 (2014).

[33] T. Oishi, K. Hagino, and H. Sagawa, Phys. Rev. C 90, 034303 (2014).
[34] G. Bertsch and H. Esbensen, Ann. Phys. 209, 327 (1991).

[35] H. Esbensen, G. F. Bertsch, and K. Hencken, Phys. Rev. C 56, 3054 (1997).

[36] K. Hagino and H. Sagawa, Phys. Rev. C 72, 044321 (2005).

[37] T. Oishi, K. Hagino, and H. Sagawa, Phys. Rev. C 82, 024315 (2010); 82, 069901(E) (2010).

[38] F. Ajzenberg-Selove, Nucl. Phys. A 490, 1 (1988) (note: several versions with the same title has been published).

[39] M. Matsuo, K. Mizuyama, and Y. Serizawa, Phys. Rev. C 71, 064326 (2005).

[40] M. Matsuo, H. Shimoyama, and Y. Ootaki, Phys. Scr. T150, 014024 (2012).

[41] K. Hagino and H. Sagawa, Phys. Rev. C 75, 021301 (2007).

[42] Y. Kikuchi, K. Katō, T. Myo, M. Takashina, and K. Ikeda, Phys. Rev. C 81, 044308 (2010).

[43] A. Bulgac and Y. Yu, Phys. Rev. Lett. 88, 042504 (2002).

[44] D. Thompson, M. Lemere, and Y. Tang, Nucl. Phys. A 286, 53 (1977).

[45] S. A. Gurvitz and G. Kalbermann, Phys. Rev. Lett. 59, 262 (1987).

[46] S. A. Gurvitz, Phys. Rev. A 38, 1747 (1988).

[47] S. A. Gurvitz, P. B. Semmes, W. Nazarewicz, and T. Vertse, Phys. Rev. A 69, 042705 (2004).

[48] V. I. Kukulin, V. M. Krasnopolsky, and J. Horáček, Theory of Resonances: Principles and Applications (Kluwer Academic Publishers, Dordrecht, Netherlands, 1989).

[49] V. Kukulin, V. Krasnopol'sky, V. Voronchev, and P. Sazonov, Nucl. Phys. A 453, 365 (1986).

[50] Y. Kobayashi and M. Matsuo, Prog. Theor. Exp. Phys. 2016, 013D01 (2016).

[51] N. S. Krylov and V. A. Fock, Zh. Éksp. Teor. Fiz. 17, 93 (1947).

[52] R. G. Winter, Phys. Rev. 123, 1503 (1961).

[53] C. A. Nicolaides and D. R. Beck, Phys. Rev. Lett. 38, 683 (1977).

[54] D. A. Dicus, W. W. Repko, R. F. Schwitters, and T. M. Tinsley, Phys. Rev. A 65, 032116 (2002).

[55] A. N. Ivanov and P. Kienle, Phys. Rev. Lett. 103, 062502 (2009).

[56] M. Peshkin, A. Volya, and V. Zelevinsky, Europhys. Lett. 107, 40001 (2014).

[57] M. Mangin-Brinet, J. Carbonell, and C. Gignoux, Phys. Rev. A 57, 3245 (1998).

[58] B. Schuetrumpf, W. Nazarewicz, and P.-G. Reinhard, Phys. Rev. C 93, 054304 (2016).

[59] M. Yamagami, J. Margueron, H. Sagawa, and K. Hagino, Phys. Rev. C 86, 034333 (2012).

[60] T. Myo, K. Katō, S. Aoyama, and K. Ikeda, Phys. Rev. C 63, 054313 (2001).

[61] L. V. Grigorenko, T. D. Wiser, K. Mercurio, R. J. Charity, R. Shane, L. G. Sobotka, J. M. Elson, A. H. Wuosmaa, A. Banu, M. McCleskey, L. Trache, R. E. Tribble, and M. V. Zhukov, Phys. Rev. C 80, 034602 (2009).

[62] G. Scamps and D. Lacroix, Phys. Rev. C 87, 014605 (2013).

[63] S. Ebata, T. Nakatsukasa, and T. Inakura, Phys. Rev. C 90, 024303 (2014).

[64] T. Nakatsukasa, K. Matsuyanagi, M. Matsuo, and K. Yabana, Rev. Mod. Phys. 88, 045004 (2016).

[65] K. Sekizawa and K. Yabana, Phys. Rev. C 88, 014614 (2013)

[66] Y. Tanimura, K. Hagino, and H. Sagawa, Phys. Rev. C 86, 044331 (2012). 